Mirela Rodrigues ${ }^{1}$ Cristine Bonfim ${ }^{2}$ José Luiz Portugal Paulo Germano de Frias ${ }^{3}$ Idê Gomes Dantas Gurgel ${ }^{4}$ Tadeu Rodrigues Costa ${ }^{1}$ Zulma Medeiros ${ }^{4}$

\section{Análise espacial da mortalidade infantil e adequação das informações vitais: uma proposta para definição de áreas prioritárias}

Spatial analysis of infant mortality and the adequacy of vital information: a proposal for defining priority areas
${ }^{1}$ Universidade Federal de Pernambuco. Av. Prof. Moraes Rego, Cidade Universitária. 50.670-901 Recife PE Brasil. mirellarod@hotmail.com ${ }^{2}$ Diretoria de Pesquisas Sociais, Fundação Joaquim Nabuco.

${ }^{3}$ Instituto de Medicina Integral Prof. Fernando Figueira.

${ }^{4}$ Centro de Pesquisas Aggeu Magalhães.
Abstract This is an ecological study that sought to assess the relationship between the spatial clustering of infant mortality and the adequacy of vital information. The adequacy of information from the Brazilian Live Birth Database (SINASC) and Mortality Database (SIM) were examined using a validated method that uses five indicators calculated by municipality and population size. Municipalities were classified as either having consolidated data, data currently being consolidated, or not having consolidated data. Voronoi polygons were generated for spatial analysis in order to minimize any proximity issues among municipalities. The local Moran index was applied to identify spatial clustering of infant mortality. It was established that $76.2 \%$ of all munic ipalities had consolidated vital data. Infant mortality clustering was seen in 34 municipalities comprising three spatial clusters. An association was also found between the adequacy of vital information and the spatial clustering of infant mortality. Geostatistical techniques proved to have predictive power to identify spatial clustering with consolidated vital information. The approach will contribute to the improvement of dat quality and can be used for planning actions seek ing to reduce infant mortality.

Key words Infant mortality, Information syste$m s$, Vital statistics, Spatial analysis
Resumo Estudo ecológico que objetivou analisar a relação entre o comportamento espacial da mortalidade infantil e a adequação das informações vitais. Para avaliar a adequação das informações sobre nascimentos (Sinasc) e óbitos (SIM) do Mi nistério da Saúde foi utilizado um método, já validado, que é constituído por cinco indicadores calculados por município, segundo o porte populacional. Os municípios foram classificados em: informações vitais consolidadas, em fase de consolidação ou não consolidadas. Na análise espacial, foram gerados os Polígonos de Voronoi para minimizar os problemas de proximidade entre os municípios, e o indice de Moran local para identificação dos agregados espaciais de mortalidade infantil. Identificou-se que 76,6\% dos municípios apresentaram informações vitais consolidadas. Houve formação de cluster para a mortalidade infantil em 34 municípios, formando três agregados espaciais. Verificou-se associação entre a adequação das informações vitais e o comportamento espacial da mortalidade infantil. As técnicas de geoestatística foram preditivas na identificação de agregados espaciais com informações vitais consolidadas. A proposta contribuirá para a melhoria da qualidade da informação e o planejamento de ações visando à redução da mortalidade infantil.

Palavras-chave Mortalidade infantil, Sistemas de informação, Estatísticas vitais, Análise espacial 


\section{Introdução}

A mensuração da mortalidade infantil (MI) é fundamental para a análise da situação de saúde de crianças, contribuindo para a avaliação de programas e para a vigilância epidemiológica dos agravos à saúde ${ }^{1,2}$.

No Brasil, o Coeficiente de Mortalidade Infantil (CMI) pode ser calculado através dos dados produzidos pelo Sistema de Informação sobre Mortalidade (SIM) e pelo Sistema de Informação sobre Nascidos Vivos (Sinasc). Desenvolvidos pelo Ministério da Saúde (MS), respectivamente nas décadas de 1970 e 1990, o SIM e o Sinasc têm abrangência nacional e vêm ampliando progressivamente sua cobertura ${ }^{3,4}$. Todavia, as disparidades regionais quanto à cobertura dos sistemas de informações vitais dificultam o uso dos dados das regiões Norte e Nordeste e em alguns estados da região centro-oeste do Brasil, não permitindo o seu cálculo a partir de dados diretos ${ }^{2,3}$.

A responsabilidade para estimar e divulgar dados referentes à MI é do Instituto Brasileiro de Geografia e Estatística (IBGE), que utiliza técnicas de mensuração indireta. Apesar de serem reconhecidas pela robustez, existem limites e incoerências relacionados a essas formas de estimação ${ }^{2}$, impossibilitando o monitoramento da MI no âmbito municipal e em especial onde existem pequenos contingentes populacionais $^{2,4}$. Por isso, o MS tem estimulado o desenvolvimento de métodos que avaliem a adequação das informações vitais e de estratégias que estimulem o uso dos dados diretos dos sistemas de informações em saúde ${ }^{5,6}$.

Em 2007, foi proposto um método para avaliar a adequação das informações vitais no âmbito municipal ${ }^{2}$, que permite identificar deficiências do SIM e Sinasc locais a partir de indicadores de mortalidade, natalidade e causa de óbito mal definida. Resultados da Pesquisa de Busca Ativa de Óbitos e Nascimentos na Região Nordeste e Amazônia Legal do Brasil apontaram para a coerência deste método: municípios classificados com informações vitais adequadas apresentaram os menores fatores de correção, enquanto os maiores foram encontrados naqueles com informações insuficientes ${ }^{6}$. Entretanto, o monitoramento da MI nos estados com informações incompletas permanece um desafio, sobretudo para o desenvolvimento de estratégias com vistas à superação ${ }^{1,5}$.

A análise espacial tem se mostrado uma alternativa promissora na identificação de áreas prioritárias para as intervenções em saúde. É sabido que a MI apresenta um comportamento não homogêneo no espaço, permitindo identificar áreas com altos e baixos riscos de morte neste grupo etário ${ }^{7}$. A formação de agregados espaciais indica a alta probabilidade de semelhança na MI entre áreas geográficas adjacentes ${ }^{8,9}$. Entretanto, nas principais bases de dados, os estudos não abordam as relações entre o comportamento espacial da MI e a adequação das informações vitais. Assim, o objetivo deste estudo foi conhecer a associação entre o comportamento espacial da MI e a adequação das informações vitais.

\section{Métodos}

Trata-se de um estudo ecológico, desenvolvido no estado de Pernambuco, localizado na Região Nordeste do Brasil, com uma área de 98.146,3 $\mathrm{Km}^{2}$ e uma população de 8.796 .448 habitantes, distribuída em 184 municípios e no distrito estadual de Fernando de Noronha.

Para avaliar a adequação das informações vitais foi utilizada a proposta de Andrade e Szwarcwald $^{2}$ que classifica as informações a partir de cinco indicadores: coeficiente de mortalidade geral padronizado por idade, desvio médio relativo do coeficiente de mortalidade geral, razão entre nascidos vivos informados e estimados, desvio médio relativo da taxa de natalidade e proporção de óbitos sem definição de causa básica.

Esses indicadores foram mensurados para cada município de acordo com o porte populacional: porte I (municípios com menos de 50 mil habitantes) e porte II (municípios com 50 mil habitantes ou mais). Foi excluído o distrito estadual de Fernando de Noronha dada a distância geográfica do Estado.

Cada um destes indicadores foi classificado em satisfatórios, não satisfatórios e deficientes. Para esta classificação foram utilizados os limites de confiança para a média dos indicadores das oito UF com informações consideradas adequadas pelos critérios da Rede Interagencial de Informações para a Saúde (Ripsa $)^{10}$. A partir do padrão alcançado por estas UF foram estabelecidos os pontos de corte ${ }^{10}$ para classificar os municípios de Pernambuco em ${ }^{11}$ :

- Informações vitais consolidadas: quando todos os indicadores foram classificados como satisfatórios.

. Informações vitais em fase de consolidação: quando pelo menos um dos indicadores foi classificado como não satisfatório e nenhum foi deficiente. 
. Informações vitais não consolidadas: quando pelo menos um dos indicadores foi classificado como deficiente.

O CMI de cada município, referente ao triênio 2006-2008, foi calculado a partir da divisão do somatório de óbitos em menores de um ano, ocorridos no triênio, pelo somatório de nascidos vivos, no mesmo período obtidos do SIM e Sinasc. Os dados foram disponibilizados pela secretaria de saúde do estado de Pernambuco.

Para análise espacial da mortalidade infantil, foram gerados os Polígonos de Voronoi (PV) a partir das sedes municipais. Considerando que nos pontos-sede concentra-se a maior parte da população, aqui serão chamados de municípios. A escolha desta técnica de geoestatística justificase por minimizar os problemas de proximidade entre os 184 municípios.

Os PV são reconhecidos por sua precisão na constituição de áreas de influência para os eventos em saúde ${ }^{12,13}$, por considerar o critério de vizinhança natural, reconstruindo os polígonos municipais a partir da influência que cada sede de município tem sobre os demais ${ }^{14}$.

Uma vez definida a nova geometria do estado, o CMI foi espacializado e empregado o índice de Moran Local para identificação de agregados espaciais. Esse índice foi calculado pelas fórmulas e qual é o CMI do município, o CMI médio dos municípios, se são vizinhos e se não são vizinhos. Caso o índice seja positivo, existem municípios vizinhos com altos (AA) ou baixos (BB) valores de CMI, caracterizando os clusters. Caso o índice seja negativo, conclui-se que um município com alto valor do coeficiente é vizinho de municípios com baixo valor de $\mathrm{CMI}(\mathrm{AB}) \mathrm{ou}$, um com baixo valor de CMI é vizinho de municípios com alto valor do coeficiente (BA), caracterizando assim os outliers. Utilizou- se o nível de significância de $5 \%$ para rejeitar a hipótese nula de que as formações $\mathrm{AA}, \mathrm{BB}, \mathrm{AB}, \mathrm{BA}$ são falsas.

Para testar a associação entre o comportamento espacial do CMI e a adequação das informações vitais foi utilizado o teste exato de Fisher, considerando um nível de significância de 5\%.

Este trabalho foi aprovado pelo Comitê de Ética em Pesquisas do Centro de Pesquisas Aggeu Magalhães, Fundação Oswaldo Cruz.

\section{Resultados}

Dos 184 municípios de Pernambuco 76,6\% apresentaram informações vitais consolidadas; $14,7 \%$ em fase de consolidação; e 9,2\% não consolida- das. Na Figura 1 observa-se que à medida que distanciam-se da capital do Estado, existem mais municípios classificados com informações em fase de consolidação ou não consolidadas.

Observou-se a formação de cluster para a mortalidade infantil em 34 municípios, formando três agregados espaciais $(\mathrm{p}<0,005)$. Destes, dois são constituídos por CMI com valores altos e um de baixo. Os agregados de CMI altos, denominado cluster alto-alto (AA), são formados por 19 municípios localizados na região central e oeste do estado. O cluster de CMI baixos é formado por 15 municípios localizados na região leste de Pernambuco, denominado cluster baixobaixo (BB). Foram identificados quatro municípios que apresentaram comportamento do coeficiente diferente dos vizinhos de primeira ordem, identificados como outliers. Dois deles apresentaram CMI baixo circundado de CMI altos (BA), e outros dois CMI alto circundado de CMI baixos $(\mathrm{AB})$ (Figura 2).

Houve associação entre o comportamento espacial da MI e a adequação das informações vitais $(p=0,013)$. Dentre os 26 municípios com informações vitais consolidadas, houve alta probabilidade de pertencer ao cluster BB $(53,8 \%)$ e baixa probabilidade de pertencer aos outliers $\mathrm{AB} /$ BA $(15,4 \%)$ (Tabela 1$)$

Entre os municípios que compõem o cluster AA, $42,1 \%$ têm informações vitais consolidadas e $31,6 \%$ em fase de consolidação ( $p=0,013)$. No cluster BB, 93,3\% apresentam informações vitais consolidadas. Entre os outliers, todos os municí-

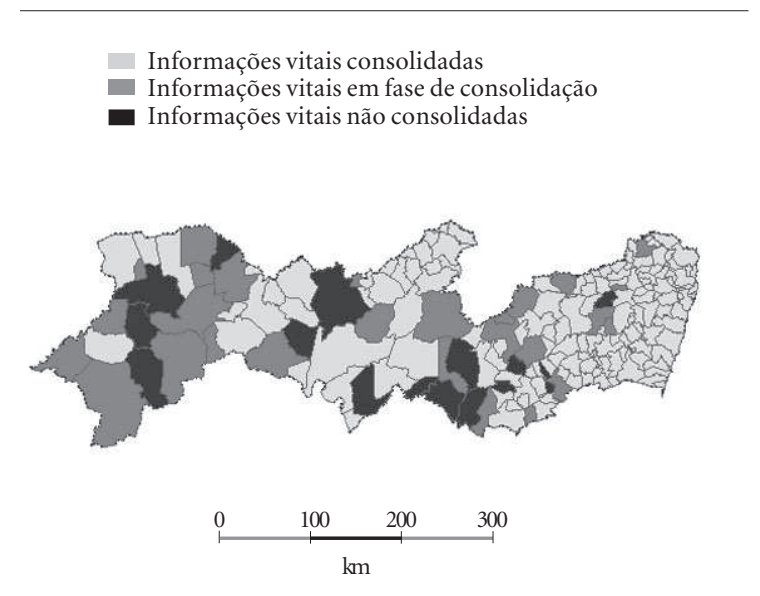

Figura 1. Distribuição espacial da adequação das informações vitais segundo municípios. Pernambuco, Brasil, 2006-2008. 
pios foram classificados como informações vitais consolidadas (Tabela 1).

Não houve significância estatística $(\mathrm{p}=0,58)$ quando os clusters AA e BB foram agregados para associar com a adequação das informações vitais.

\section{Discussão}

A classificação das informações vitais em Pernambuco aqui encontradas ratificam os avanços na cobertura e regularidade do SIM e Sinasc no estado $^{2,10,11}$. Recentes estudos desenvolvidos em Pernambuco, que tiveram como propósito avaliar a adequação dessas informações, evidencia- ram a contínua melhoria na qualidade das informações produzidas por estes sistemas ${ }^{10,11}$.

O comportamento espacial da MI apresentou-se conforme o observado no mundo ${ }^{7,9,15-19} \mathrm{e}$ no Brasi ${ }^{20-26}$. No estado de Pernambuco, a MI se distribuiu de forma heterogênea, formando agregados espaciais de alto e baixo risco para o óbito, evidenciando que este evento não ocorre de forma aleatória entre os municípios. A ocupação espacial da população sob risco de morte antes do primeiro ano de vida pode ser determinada por aspectos geográficos e socioeconômicos, permitindo refletir sobre sua dependência espacial ${ }^{22,24}$.

A associação encontrada entre o comportamento espacial da MI e a adequação das informações vitais evidencia a predição do método
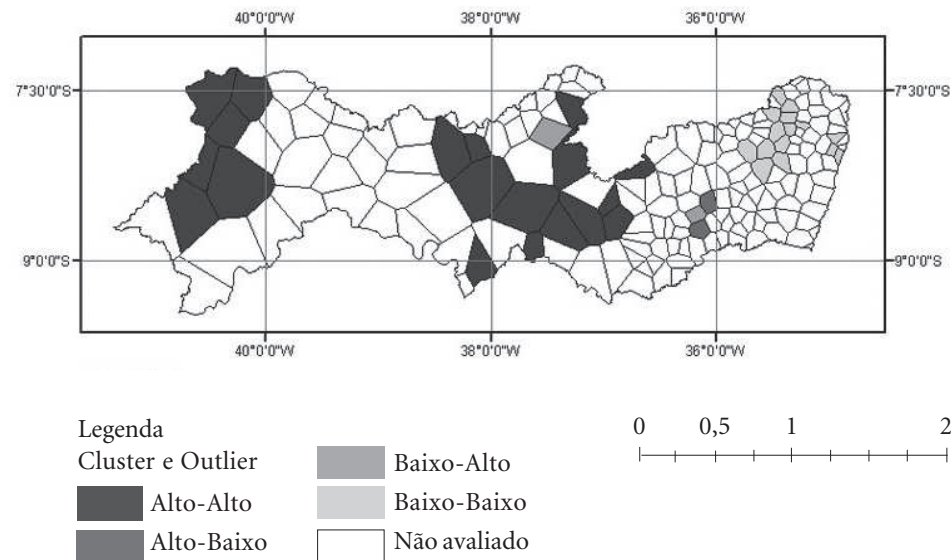

$\begin{array}{llll}0 & 0,5 & 1 & 2 \text { graus decimais }\end{array}$

2006-2008

Tabela 1. Adequação das informações vitais segundo diferentes tipos de formações espaciais para a mortalidade infantil. Pernambuco, Brasil, 2006-2008.

\begin{tabular}{lccrr}
\hline Adequação das Informações Vitais & Alto-Alto & Baixo-Baixo & Alto-Baixo/Baixo-Alto & Total \\
\hline Consolidada & 8 & 14 & 4 & 26 \\
\% Linha & 30,8 & 53,8 & 15,4 & 100 \\
\% Coluna & 42,1 & 93,3 & 100 & 68,4 \\
Em fase de consolidação & 6 & - & - & 6 \\
\% Linha & 100 & - & - & 100 \\
\% Coluna & 31,6 & - & - & 15,8 \\
Não consolidada & 5 & 1 & - & 6 \\
\% Linha & 83,3 & 16,7 & - & 100 \\
\% Coluna & 26,3 & 6,7 & 4 & 15,8 \\
Total & 19 & 15 & & 38
\end{tabular}

Fisher $=12,62 ; p=0,013$ 
que avaliou as informações sobre nascimentos e óbitos. A maioria dos municípios cuja MI foi semelhante a de seus vizinhos foi classificada como informações vitais consolidadas. Destaque para aqueles que compuseram o cluster $\mathrm{BB}$, indicando maior probabilidade em ter informações adequadas.

A maioria dos municípios que compõem o cluster AA apresentou informações consolidadas, permitindo identificar aqueles com dados confiáveis e cuja MI encontra-se em altos níveis. Neste cluster, aproximadamente $26 \%$ foram classificados como informações vitais não consolidadas. Entre eles, encontram-se municípios de pequeno porte populacional e com baixos Índices de Desenvolvimento Humano (IDH).

A tentativa de agregar os clusters AA com BB e verificar a associação com a adequação das informações vitais provocou a perda da significância estatística, sugerindo que estas duas formas de agregação espacial da MI não são homogêneas, não devendo ser analisadas conjuntamente.

Os municípios que compuseram o grupo $\mathrm{AB} /$ BA foram classificados como consolidados para as informações vitais e todos têm população inferior a 50.000 habitantes. Assume-se que o resultado pode ter sido influenciado pelos pequenos números, tendo em vista a existência de apenas quatro municípios classificados como outliers. As autoras do método de avaliação da adequação das informações vitais ${ }^{2}$ adotaram critérios mais flexíveis e amplos para os municípios menores de 50 mil habitantes em virtude da flutuação dos indicadores existente em pequenos contingentes populacionais, podendo gerar o uso de informações menos precisas, comprometendo os indicadores de saúde mensurados pelo modo direto ${ }^{2}$.

Todos os municípios classificados como informações vitais em fase de consolidação pertenceram ao cluster AA, evidenciando altos níveis de MI. Este grupo de municípios representa aqueles que estão em fase de transição da qualidade da informação de não consolidada para a consolidada, e por este motivo de difícil reflexão.

O MS tem desenvolvido ações e incentivado estratégias para a melhoria da cobertura e da qualidade das informações vitais ${ }^{4}$, enfatizando a importância das estimativas do CMI pelo método direto e renovando o interesse pelas informações vitais de registro contínuo ${ }^{4-6}$. Entre as ações do MS citam-se $\mathrm{s}^{5}$ a divulgação dos dados por município em duas diferentes formas de acesso (CD-ROM e na internet); o investimento em consultores técnicos nos estados para a qualificação das informações vitais; a inclusão de metas rela- cionadas ao SIM e ao Sinasc à Programação Pactuada e Integrada (PPI); a elaboração do Sistema de Monitoramento de Indicadores Relacionados à Mortalidade Infantil (MONITORIMI) e o estudo de busca ativa de óbitos e nascimentos nas Regiões Norte e Nordeste ${ }^{6}$.

Esta última estratégia evidenciou que os fatores de correção para o cálculo da MI, a partir dos dados diretos do SIM e Sinasc, apresentam variação de 1,08 a 3,27, sendo menores para os municípios de grande porte, localizados na Amazônia Legal, com informações vitais satisfatórias, e os maiores para os municípios pequenos, situados no Nordeste do país e cujas informações vitais são inadequadas. O estado de Pernambuco apresentou um fator de correção de 1,20 para o cálculo do CMI, destacando-se como o menor da Região Nordeste, junto ao estado de Sergipe, e semelhante ao Brasil $(1,22)^{6}$.

Identificar as irregularidades locais nos sistemas de informação proporciona a melhora da qualidade das estatísticas vitais ${ }^{13,27}$ e, assim, a estimação da mortalidade infantil. Embora a adequação das informações não signifique cobertura absoluta dos sistemas, o cálculo da mortalidade infantil pode ser realizado pelo método direto para os municípios com informações vitais consolidadas, utilizando os dados disponíveis no SIM e Sinasc locais². Assim, torna-se possível monitorar a MI, identificando seus diferenciais, áreas de maior risco e a ampla compreensão dos seus determinantes ${ }^{28}$. Com informações qualificadas é possível desenvolver ações direcionadas para reduzir as iniquidades da MI, atingindo grupamentos populacionais expostos a riscos aumentados.

A utilização de técnicas de geoestatística mostrou-se preditiva na identificação de agregados espaciais com informações vitais consolidadas, confirmando a precisão do método de adequação das informações sobre nascimentos e óbitos. Ademais, a junção destas duas estratégias mostrou-se um importante eixo para as intervenções em saúde na medida em que permitirá identificar os municípios com distintas realidades:

(I) Aqueles que apresentam informações vitais em padrões confiáveis e altos níveis de MI, para que as intervenções sejam direcionadas, prioritariamente, sobre os determinantes das mortes infantis;

(II) Aqueles que apresentam informações vitais em padrões confiáveis e baixos níveis de MI, permitindo investigar quais os fatores que influenciam positivamente na magnitude deste indicador e direcionar as intervenções aos determinantes do componente assistência neonatal; 
(III) Aqueles que apresentam informações vitais em padrões não confiáveis e altos níveis de MI, apontará que a melhor ação será adequar a cobertura e a regularidade dos sistemas, acrescida a intervenções direcionadas aos determinantes dessas mortes;

(IV) Aqueles que apresentam informações vitais em padrões não confiáveis e baixos níveis de MI, os quais precisarão de intervenções tanto para a adequação das informações vitais quanto para os determinantes da MI.

Estudos que relacionem o comportamento espacial da MI com a adequação das informações vitais podem aumentar o poder de discriminação de intervenções necessárias para melhorar a qualidade dos dados dos sistemas de informações sobre óbitos e nascimentos, e direcionar as ações sobre os determinantes sociais e assistenciais da MI.

\section{Colaboradores}

M Rodrigues, Z Medeiros, TR Costa, IGD Gurgel, PG Frias, C Bonfim e JL Portugal participaram igualmente de todas as etapas de elaboração do artigo. 


\section{Referências}

1. Torres C, Mujica OJ. Health, equity, and the millennium development goals. Rev Panam Salud Pública 2004; 15(6):430-439.

2. Andrade CLT, Szwarcwald CL. Desigualdades sócio-espaciais da adequação das informações de nascimentos e óbitos do Ministério da Saúde, Brasil, 2000-2002. Cad Saude Publica 2007; 23(5):1207-1216.

3. Mello-Jorge MH, Laurenti R, Gotlieb SLD. Análise da qualidade das estatísticas vitais brasileiras: a experiência de implantação do SIM e do SINASC. Cien Saude Colet 2007; 12(3):643-654.

4. Frias PG, Pereira PMH, Andrade CLT, Szwarcwald CL. Sistema de Informações sobre Mortalidade: estudo de caso em municípios com precariedade dos dados. Cad Saude Publica 2008; 24(10):2257-2266.

5. Frias PG, Szwarcwald CL, Lira PIC. Estimação da mortalidade infantil no contexto de descentralização do Sistema Único de Saúde (SUS). Rev. Bras. Saúde Matern. Infant. 2011; 11(4):463-470.

6. Szwarcwald CL, Morais Neto OL, Frias PG, Souza Junior PRB, Escalante JJC, Lima RB, Viola RC. Busca ativa de óbitos e nascimentos no Nordeste e na Amazônia Legal: Estimação da mortalidade infantil nos municípios brasileiros. In: Ministério da Saúde (MS). Secretaria de Vigilância em Saúde. Departamento de Análise de Situação de Saúde. Saúde Brasil 2010: uma análise da situação de saúde e de evidências selecionadas de impacto de ações de vigilância em saúde. Brasilia: MS; 2011.

7. Chin B, Montana L, Basagaña. Spatial modeling of geographic inequalities in infant and child mortality across Nepal. Health \& Place 2011; 17(4):929-936.

8. Grady SC, Enander H. Geographic analysis of low birthweight and infant mortality in Michigan using automated zoning methodology. Int J Health Geogr 2009; 8:10.

9. Balk D, Pullum T, Storeygard A, Greenwell F, Neuman M A. Spatial Analysis of Childhood Mortality in West Africa. Popul Space Place 2004; 10(3):175-216.

10. Duczmal LH, Moreira GJP, Burgarelli D, Takahashi RHC, Magalhães FCO, Bodevan EC, Voronoi distance based prospective space-time scans for point data sets: a dengue fever cluster analysis in a southeast Brazilian town. Int J health geogr 2011; 10:29.

11. Wakamatsu M, Kikuchi A, Tamaru S, Ono K, Horikoshi T, Takagi K, Ogiso Y, Tanemura M. Voronoi diagram description of the maternal surface of the placenta: Preliminary report. J Obstet Gynaecol Res 2011; 37(7):792-799.

12. Silva RR, Bacha CJC. Polígonos de Voronoi como alternativa aos problemas das Áreas Mínimas Comparáveis: uma análise das mudanças populacionais na Região Norte do Brasil. Rev. bras. estud. popul. 2011; 28(1):133-151

13. Frias PG, Pereira PMH, Andrade CLT, Lira PIC, Szwarcwald CL. Avaliação da adequação das informações de mortalidade e nascidos vivos no Estado de Pernambuco, Brasil. Cad Saude Publica 2010; 26(4):671-681.
14. Rodrigues M, Bonfim C, Frias PG, Braga C, Gurgel IGD, Medeiros Z. Diferenciais na adequação das informações de eventos vitais nos municípios de Pernambuco, 2006-2008. Rev Bras Epidemiol 2012; 15(2):275-284.

15. Danke K, Blecher C, Bardehle D, Cremer D, Razum O. Small area analysis of infant mortality in Bielefeld with special consideration of the migration status of parents, 2000-2006. Gesundheitswesen 2008; 70(11):624-630.

16. Alvarez G, Lara F, Harlow SD, Denman C. Infant mortality and urban marginalization: a spatial analisys of their relationship in a medium sized city in northwest Mexico. Rev Panam Salud Publica 2009; 26(1):31-38.

17. Tottrup C, Tersbol BP, Lindeboom W, Meyrowitsch D. Putting child mortality on a map: towards an understanding of inequity in health. Trop Med Int Health 2009; 14(6):653-662.

18. Sohel N, Vahter M, Ali M, Rahman M, Rahman A Streatfield PK, Kanaroglou PS, Persson LA. Spatial patterns of fetal loss and infant death in an arsenicaffected area in Bangladesh. Internacional Int $J$ Health Geogr 2010; 9:53

19. Kazembe LN, Mpeketula PM. Quantifying spatial disparities in neonatal mortality using a structured additive regression model. PLoS One 2010; 5(6):10.

20. Silva SLC, Fachel JMG, Kato SK, Bassanesi SL. Visualização dos padrões de variação da taxa de mortalidade infantil no Rio Grande do Sul, Brasil: comparação entre as abordagens Bayesiana Empírica e Totalmente Bayesiana. Cad Saude Publica 2011; 27(7): 1423-1432.

21. Gonçalves AC, Costa MCN, Braga JU. Análise da distribuição espacial da mortalidade neonatal e de fatores associados, em Salvador, Bahia, Brasil, no período 2000-2006. Cad Saude Publica 2011; 27(8): 1581-1592.

22. Bezerra Filho JG, Kerr LRS, Miná DL, Barreto ML Distribuição espacial da taxa de mortalidade infantil e principais determinantes no Ceará, Brasil, no período 2000-2002. Cad Saude Publica 2007; 23(5): 1173-1185.

23. Nascimento LFC, Batista GT, Dias, NW, Catelani CS, Becker D, Rodrigues L. Análise espacial da mortalidade neonatal no Vale do Paraíba, 1999 a 2001. Rev Saude Publica 2007; 41(1):94-100.

24. Andrade CLT, Szwarcwald CL. Análise espacial da mortalidade neonatal precoce no Município do Rio de Janeiro, 1995-1996. Cad Saude Publica 2001; 17(5): 1199-1210.

25. Oliveira GS, Lima MCBM, Lyra CO, Oliveira AGRC Ferreira MAF. Desigualdade espacial da mortalidade neonatal no Brasil: 2006-2010. Cien Saude Colet 2013; 18(8):2431-2441

26. Garcia LP, Santana LR. Evolução das Desigualdades socioeconômicas na mortalidade infantil no Brasil, 1993-2008. Cien Saude Colet 2011; 16(9):37173728 . 
27. Almeida MF, Alencar GP, Novaes HMD, Ortiz LP. Sistemas de informação e mortalidade perinatal: conceitos e condições de uso em estudos epidemiológicos. Rev Bras Epidemiol 2006; 9(1):56-68.

28. Muller M, Drack G, Schindler C, Bucher HU. Liveborn and stillborn very low birthweight infants in Switzerland: comparison between hospital based birth registers and the national birth register. Swiss Med Wkly 2005; 135(29-30):433-439.

Artigo apresentado em 26/07/2013

Aprovado em 22/09/2013

Versão final apresentada em 26/09/2013 